专家推介: 本文研究了真空热处理、紫外臭氧辐照及氢气热处理 3 种工艺对制备防离子反馈膜层后的 MCP 进 行除碳处理的结果, 并通过 AES 能谱仪对 MCP 表面的碳成分进行了分析, 得出氢气热处理工艺对 MCP 的除 碳效果最好, 增益提升最大的结论。

\title{
防离子反馈微通道板表面碳污染去除的试验研究
}

杨晓军 ${ }^{1,2}$, 李 丹 ${ }^{1,2}$, 乔 凯 ${ }^{1,2}$, 师宏立 ${ }^{1,2}$, 郝子恒 ${ }^{1,2}$, 张 妮 ${ }^{1,2}$, 刘 峰 ${ }^{1,2}$, 刘旭川 ${ }^{1,2}$ (1. 微光夜视技术重点实验室, 陕西 西安 710065；2. 昆明物理研究所, 云南 昆明 650223)

\begin{abstract}
摘要: 针对带防离子反馈膜的微通道板 ( $\mathrm{MCP}$ ) 普遍存在的 $\mathrm{C}$ 污染现象, 开展了真空热处理、紫外臭 氧辐照及氢气热处理试验, 对各个试验结果进行了 MCP 电性能测试及俄歇电子能谱( AES) 研究, 着重分析了 $\mathrm{MCP}$ 表面 $\mathrm{C}$ 含量及 $\mathrm{MCP}$ 性能随不同试验方法的变化趋势。试验结果表明, C 含量的降 低对 $\mathrm{MCP}$ 性能的提升具有显著作用, 真空热处理对 $\mathrm{C}$ 的分解作用甚微, 紫外臭氧辐照及氢气热处理 均可以较为彻底地去除 $\mathrm{MCP}$ 表面 $\mathrm{C}$ 污染, 并且氢气热处理对 $\mathrm{MCP}$ 增益性能的提升作用显著, 效率 最高。
\end{abstract}

关键词: C 污染; 防离子反馈 $\mathrm{MCP}$; 紫外辐照; 氢气处理

中图分类号：TN223 文献标志码：A 文章编号：1001-8891(2020)08-0747-05

\section{Experimental Study of Carbon Pollution Removal from Microchannel Plate with Ion Barrier Film}

YANG Xiaojun $^{1,2}$, LI Dan ${ }^{1,2}$, QIAO Kai ${ }^{1,2}$, SHI Hongli ${ }^{1,2}$, HAO Ziheng ${ }^{1,2}$, ZHANG Ni ${ }^{1,2}$, LIU Feng ${ }^{1,2}$, LIU Xuchuan ${ }^{1,2}$ (1. Science and Technology on Low-Light-Level Night Vision Laboratory, Xi'an 710065, China;

2. Kunming Institute of Physics, Kunming 650223, China)

\begin{abstract}
To investigate the common C pollution phenomenon of the microchannel plate (MCP) with an ion barrier film, we conducted vacuum heating, ultraviolet irradiation, and hydrogen heating tests. Auger electron spectroscopy and MCP electrical performance test are performed on each test result. Furthermore, the trends of MCP C content and MCP performance are analyzed using different methods. The analysis results show that the reduction in $\mathrm{C}$ content significantly improved MCP performance. The vacuum heating process does not significantly affect the decomposition of $\mathrm{C}$; however, the UV irradiation and hydrogen heating processes can completely remove MCP C pollution. Finally, hydrogen heating significantly improved the MCP gain performance and resulted in the highest efficiency.
\end{abstract}

Key words: $\mathrm{C}$ pollution, MCP with ion barrier film, UV irradiation, hydrogen heating process

\section{0 引言}

第三代微光夜视技术以高灵敏度负电子亲和势 $\mathrm{GaAs}$ 光电阴极和高增益、低噪声、长寿命、带 $\mathrm{Al}_{2} \mathrm{O}_{3}$ 防离子反馈膜的 MCP (microchannel plate) 为主要特 色 $^{[1]}$ 。为了提高三代微光像增强器的使用寿命, 保持 其高灵敏度特性, 国内外大多研究者 ${ }^{[2-4]}$ 认为, 采用带 防离子反馈膜的 MCP 是最有效的技术手段。

但在防离子反馈 $\mathrm{MCP}$ 的使用过程中发现, MCP
表面普遍存在严重的碳元素污染（以下简称 $\mathrm{C}$ 污染） 现象。C 污染会造成次级电子发射体表面势垒增加, 通道内表面二次电子发射系数变小, 发射层和导电层 之间电阻变大 ${ }^{[5]}$, 引起 $\mathrm{MCP}$ 电子增益、体电阻和暗 电流发生有害变化, 降低了三代微光像增强器的寿 命、信噪比、亮度增益和祄度等性能 ${ }^{[6]}$, 导致像管质 量不稳定以至失效。因此, 如何彻底去除 $\mathrm{C}$ 污染是高 性能防离子反馈 MCP 一直研究的重点课题, 其对提 高三代微光像增强器的质量和寿命具有重要意义。 
本文基于 $\mathrm{Al}_{2} \mathrm{O}_{3}$ 防离子反馈 $\mathrm{MCP}$ 结构特性及成 膜工艺机理, 针对防离子反馈 $\mathrm{MCP}$ 表面的 $\mathrm{C}$ 污染问 题, 进行了防离子反馈 MCP 真空热处理、紫外臭氧 辐照处理去 $\mathrm{C}$ 试验, 并首次开展氢气热处理去除 $\mathrm{C}$ 污 染试验, 对各个试验结果进行了 MCP 电性能测试及 AES (Auger electron spectroscopy) 研究, 详细分析了 不同处理方式对防离子反馈 $\mathrm{MCP}$ 表面 $\mathrm{C}$ 含量及 $\mathrm{MCP}$ 性能的影响, 为低 $\mathrm{C}$ 污染防离子反馈 $\mathrm{MCP}$ 的制作提 供理论基础和技术支撑。

\section{1 防离子反馈 MCP 表面 C 污染的来源分析}

防离子反馈 $\mathrm{MCP}$ 表面 $\mathrm{C}$ 污染来源主要有两个方 面。一是防离子反馈膜成膜过程的引入。由于 $\mathrm{MCP}$ 具有多孔结构特性, 要直接在其多孔表面上镀制一层 超薄、连续、致密的 $\mathrm{Al}_{2} \mathrm{O}_{3}$ 膜难以实现, 因此在镀制 $\mathrm{Al}_{2} \mathrm{O}_{3}$ 膜前, 需要在 $\mathrm{MCP}$ 输入面涂敷一层有机膜作为 载体, 然后采用电子束蒸发、二极骤冷直流离子浌射 等方法在有机膜上沉积一层 $\mathrm{Al}_{2} \mathrm{O}_{3}$ 膜, 最后加温分解 去除有机膜 ${ }^{[7-8]}$, 得到带有 $\mathrm{Al}_{2} \mathrm{O}_{3}$ 防离子反馈膜的 $\mathrm{MCP}$ 。该有机膜主要由硝化纤维素、助溶剂、增塑剂 等有机物质组成, 温度超过 $120^{\circ} \mathrm{C}$ 时碳化, 超过 $360^{\circ} \mathrm{C}$

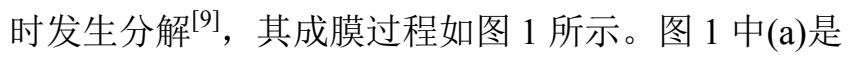
待处理 MCP, (b) 是涂敷有机膜后的 MCP, (c) 是在有 机膜表面镀制防离子反馈膜后的 MCP, (d) 是去除有 机膜后的防离子反馈 $\mathrm{MCP}$ 。由于制作过程中有机膜与 $\mathrm{MCP}$ 表面直接贴合, 若有机膜分解不彻底, MCP 膜 层表面及 $\mathrm{MCP}$ 通道内壁就会存在 $\mathrm{C}$ 残留, 从而造成 $\mathrm{MCP}$ 表面 $\mathrm{C}$ 污染, 这是 $\mathrm{MCP}$ 被 $\mathrm{C}$ 污染的一个重要来 源; 二是 MCP 自身制备过程中的引入。 MCP 的制作 工艺复杂、周期较长, 在制作过程中, 需要多次用有 机溶剂进行清洗或脱水处理, 有机溶剂的使用成为另 一个重要的 $\mathrm{C}$ 污染来源。此外, 空气中的游离 $\mathrm{C}$, 所 有的 $\mathrm{C}-\mathrm{H}$ (碳氢化合物), $\mathrm{CO}, \mathrm{CO}_{2}$ 都可以对微通道 板表面形成 $C$ 污染 ${ }^{[10]}$ 。

\section{2 试验}

\section{1 试验方法}

试验选取同种规格、同种材料、同种工艺制作的 $\mathrm{MCP}$ 若干, 采用相同工艺对所有样品进行有机膜涂敷 及 $\mathrm{Al}_{2} \mathrm{O}_{3}$ 膜镀制, 并分别对样品进行编号待用。

1) 选取 MCP 样品两片, 分别在无油真空炉中进 行 $400^{\circ} \mathrm{C}$ 保温 $2 \mathrm{~h}$ 及保温 $15 \mathrm{~h}$ 试验, 以验证真空处理 时间对 MCP 表面 C 含量的影响。试验结束后对样品 进行电性能测试及 AES 深度分析, 整个试验过程真 空度不低于 $4 \times 10^{-5} \mathrm{~Pa}$ 。

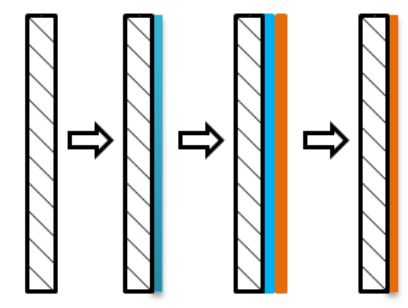
(a)
(c)
(d)

(a) 待处理 MCP; (b) 带有有机膜的 MCP; (c) 在有机膜表面镀 制防离子反馈膜后的 MCP; (d) 去除有机膜后的防离子反馈 MCP (a) Pending MCP;(b)MCP with organic film; (c) MCP with ion barrier film coated on the surface of organic film; (d) MCP with ion barrier film after removing the organic film

\section{图 1 防离子反馈 $\mathrm{MCP}$ 成膜过程示意图}

Fig.1 Schematic diagram of the formation process of MCP with ion barrier film

2) 选取经真空烘烤 $\left(400^{\circ} \mathrm{C}, 2 \mathrm{~h}\right)$ 后的 MCP 样 品 8 片, 平均分成 4 组, 在 NOVASCAN PSD-UV8 型紫外臭氧清洗仪中置于循环氧气的密闭容器内接 受 UV 辐照, 紫外灯尺寸为 8 英寸 $\times 8$ 英寸, 4 组辐 照时间分别为 $30 \mathrm{~h}, 40 \mathrm{~h}, 50 \mathrm{~h}, 100 \mathrm{~h}$ 。试验结束后, 分别对 4 组样品进行电性能测试及 AES 深度分析, 以分析经过紫外辐照后 $\mathrm{MCP}$ 性能的变化情况。

3) 选取经真空烘烤 $\left(400^{\circ} \mathrm{C}, 2 \mathrm{~h}\right)$ 后的 $\mathrm{MCP}$ 样 品两片, 在外热卧式石英管氢气炉中进行氢气热处 理, 试验温度为 $400^{\circ} \mathrm{C}$, 保温时间 $1 \mathrm{~h}$ 。试验结束后, 分别对两片样品进行电性能测试及 AES 深度分析, 以分析经过氢气热处理后 $\mathrm{MCP}$ 性能的变化情况。

\section{2 试验装置}

本文中样品深度剖面的 AES 成分分布测试是在 Thermo Scientific 俄歇电子能谱仪中进行的, 配合氩 离子溅射剥离技术, 以获得元素含量随溅射时间的关 系曲线。样品体电阻、增益等电性能测试装置均为 MCP 综合性能检测装置, 测试系统原理如图 2 所示。

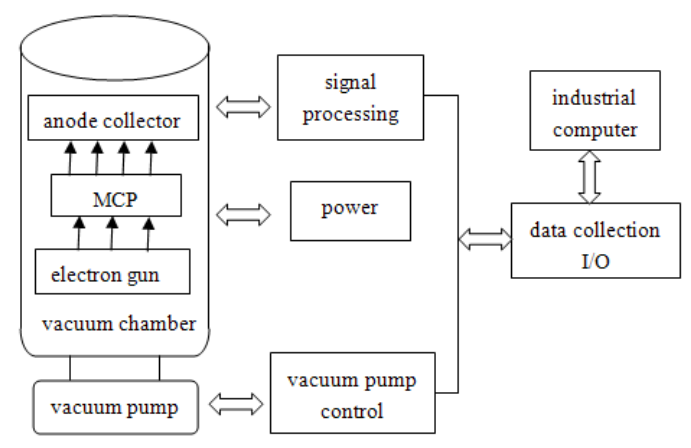

图 $2 \mathrm{MCP}$ 性能测试系统图

Fig.2 Schematic diagram of MCP performance test system

\section{3 试验结果与讨论}




\section{1 真空热处理试验}

真空高温烘烤去除有机膜是目前最常用的去膜 工艺, 其原理是在高温持续加热下, 将有机高分子材 料分解成碳化物、氮化物、氧化物、碳氢化合物及水 等物质逸出, 从而去除有机膜。表 1 为试验样品电性 能测试结果。

从表 1 可以看出, 经过长时间的高温真空烘烤, 面电阻 $\left(R_{\mathrm{a}}\right)$ 、体电阻 $\left(R_{\mathrm{b}}\right)$ 变化十分微弱, 说明在这 个过程中 MCP 表面电极及通道内表面状态没有发生 明显变化, 同样增益 $(G)$ 的变化幅度很小, 侧面反 映出, $2 \mathrm{~h}$ 的烘烤时间和 $15 \mathrm{~h}$ 的烘烤时间对有机膜的 分解效果影响很小, 对样品进行深度剖面 AES 成分 分布测试, 如图 3 所示。由于本文旨在对 MCP 表面 C 污染的去除进行试验研究, 因此在元素成分分析中, 着重对 C 元素含量的变化进行了讨论, 对其余元素不 做讨论。

图 3(a)中, 在蚀刻前 $10 \mathrm{~s}$ 内, C 元素含量迅速下 降至 10\%左右, 之后随着蚀刻时间的增加, C 元素含 量没有明显的变化趋势; 而图 3(b)中, MCP 表面 C 污染更为严重, 在蚀刻过程中 C 含量最高达到 $30 \%$ 以 上。两图中, 在蚀刻进行至 $150 \mathrm{~s}$ 之后, $\mathrm{Al}$ 元素含量 已接趋近于 0 , 而 C 元素的含量大约为 $10 \%$, 且变化 十分缓慢, 说明 MCP 通道内部存在 C 污染现象。另 一方面, 从图中可以看出, 烘烤时间的增加并没有使 C 元素的含量呈现规律的下降趋势, 最终 MCP 通道 内 C 含量差别不大, 可见, 真空环境下有机膜的分解 不够彻底。

\section{2 紫外臭氧辐照试验}

紫外 (Ultraviolet) 臭氧分解技术又称紫外光清洗 技术, 是利用有机化合物的光敏氧化作用去除黏附在 材料表面上的有机物质。国内北京理工大学的闰金良 等人 ${ }^{[11]}$ 曾对 MCP 进行了 $15 \mathrm{~h}$ 的紫外照射清洗试验, 照射后对 MCP 进行了电性能测试, 结果表明 MCP 的 体电阻下降 5\%左右, 电子增益增加 10\%左右。但他 并未对试验前后的 MCP 表面 C 元素含量进行分析和 表征, 试验条件也不是十分明确。本次试验将清洗时 间分为 $30 \mathrm{~h}, 40 \mathrm{~h}, 50 \mathrm{~h}, 100 \mathrm{~h}$, 以研究紫外清洗时 间对 MCP 性能的影响, 同时从 MCP 表面元素成分分 布特性对试验进行了更深层面的研究。表 2 为试验样 品电性能测试结果。

从表 2 可以看出, MCP 的体电阻与面电阻在紫外 辐照前后变化均不大, 说明了此条件下紫外辐照对 MCP 表面的电极不会造成氧化; 从增益情况来看, 紫 外照射 $30 \mathrm{~h}$ 和 $40 \mathrm{~h}$ 后增益变化十分微小, 说明此条件 下 C 的分解很微弱, 而当照射时间达到 $50 \mathrm{~h}$ 时增益增 加 5\%左右, 达到 $100 \mathrm{~h}$ 后增益增加 10\%左右, 可见, 照射时间达到 $50 \mathrm{~h}$ 以后 MCP 的增益才有较为显著的 增加, MCP 表面 C 残留才能进行较为快速的分解脱 附, 图 4 是 $50 \mathrm{~h}$ 紫外臭氧分解试验后样品的深度剖面 AES 成分分布。

从图 4 中可以看出, 蚀刻时间为 0 时, $\mathrm{C}$ 元素含 量为 $15.97 \%$; 随着蚀刻时间的增加, C 元素含量迅速 减少，在蚀刻进行到 $20 \mathrm{~s}$ 左右时 $\mathrm{C}$ 元素含量降为 0 , 可见 C 元素只存在于防离子反馈膜的表面, 蚀刻之初 检测到的 C 元素可能是 MCP 表面吸附空气中的 $\mathrm{CO}_{2}$ 所致。同时, 紫外照射清洗前后 MCP 表面含有的 $\mathrm{Al}$

表 1 真空热处理 MCP 参数测试结果

Table 1 MCP test results after vacuum heating process

\begin{tabular}{|c|c|c|c|c|c|c|}
\hline \multirow{2}{*}{ Number } & \multicolumn{2}{|c|}{$R_{\mathrm{a}} / \mathrm{M} \Omega$} & \multicolumn{2}{|c|}{$R_{\mathrm{b}} /(800 \mathrm{~V}) / \mathrm{M} \Omega$} & \multicolumn{2}{|c|}{$G(800 \mathrm{~V})$} \\
\hline & $2 \mathrm{~h}$ & $15 \mathrm{~h}$ & $2 \mathrm{~h}$ & $15 \mathrm{~h}$ & $2 \mathrm{~h}$ & $15 \mathrm{~h}$ \\
\hline 01\# & 67 & 65 & 121 & 117 & 1024 & 1033 \\
\hline 02\# & 87 & 83 & 136 & 139 & 876 & 885 \\
\hline
\end{tabular}

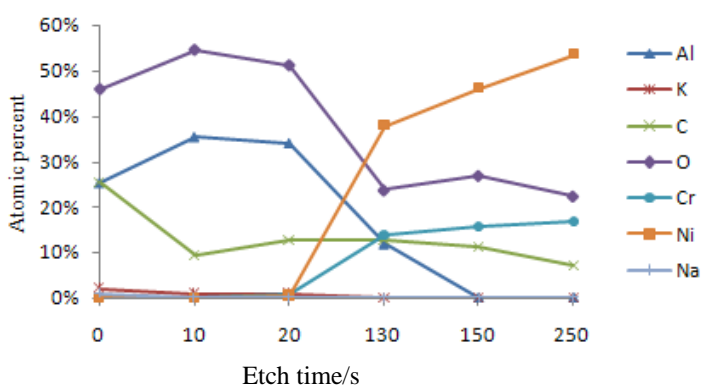

(a) $2 \mathrm{~h}$

图 3 不同真空烘烤时间后的 MCP 深度剖面分析

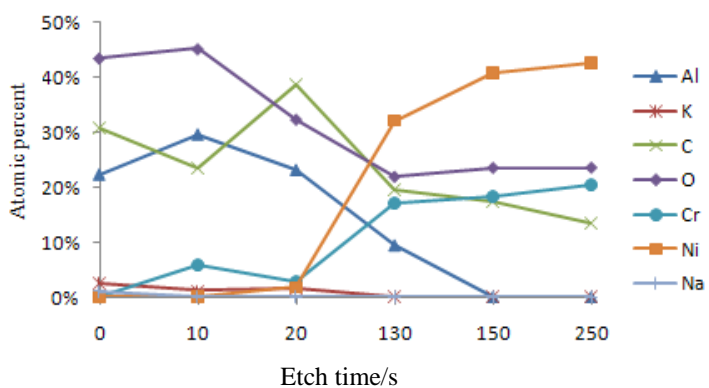

(b) $15 \mathrm{~h}$

Fig.3 Analysis of MCP depth profiles at different vacuum heating time 


\begin{tabular}{|c|c|c|c|c|c|c|c|}
\hline \multirow[b]{2}{*}{ Number } & \multirow{2}{*}{$\begin{array}{l}\text { Irradiation } \\
\text { time }\end{array}$} & \multicolumn{2}{|c|}{$R_{\mathrm{a}} / \mathrm{M} \Omega$} & \multicolumn{2}{|c|}{$R_{\mathrm{b}} /(800 \mathrm{~V}) / \mathrm{M} \Omega$} & \multicolumn{2}{|c|}{$G(800 \mathrm{~V})$} \\
\hline & & $\begin{array}{c}\text { Before } \\
\text { irradiation }\end{array}$ & $\begin{array}{c}\text { After } \\
\text { irradiation }\end{array}$ & $\begin{array}{c}\text { Before } \\
\text { irradiation }\end{array}$ & $\begin{array}{c}\text { After } \\
\text { irradiation }\end{array}$ & $\begin{array}{c}\text { Before } \\
\text { irradiation }\end{array}$ & $\begin{array}{c}\text { After } \\
\text { irradiation }\end{array}$ \\
\hline $3 \#$ & $100 \mathrm{~h}$ & 78 & 76 & 139 & 122 & 2200 & 2456 \\
\hline $4 \#$ & $100 \mathrm{~h}$ & 56 & 60 & 170 & 166 & 3339 & 3699 \\
\hline $5 \#$ & $50 \mathrm{~h}$ & 78 & 78 & 152 & 161 & 2758 & 2902 \\
\hline $6 \#$ & $50 \mathrm{~h}$ & 124 & 129 & 130 & 128 & 2862 & 3001 \\
\hline $7 \#$ & $40 \mathrm{~h}$ & 66 & 65 & 102 & 105 & 2888 & 2900 \\
\hline $8 \#$ & $40 \mathrm{~h}$ & 82 & 80 & 115 & 119 & 3199 & 3227 \\
\hline $9 \#$ & $30 \mathrm{~h}$ & 75 & 73 & 108 & 115 & 2572 & 2597 \\
\hline $10 \#$ & $30 \mathrm{~h}$ & 88 & 89 & 133 & 135 & 2352 & 2380 \\
\hline
\end{tabular}

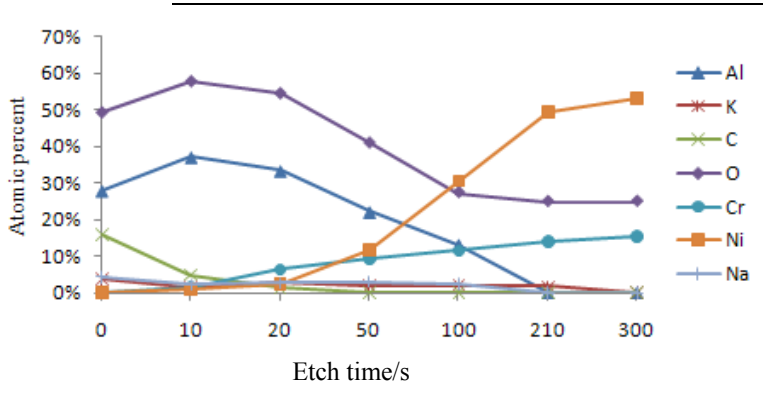

图 4 紫外臭氧辐照 $50 \mathrm{~h}$ 后 MCP 深度剖面分析

Fig.4 Analysis of MCP depth profiles after UV irradiation at $50 \mathrm{~h}$ 元素与 $\mathrm{O}$ 元素均无明显变化, 可见此条件不会对 $\mathrm{MCP}$ 表面的防离子反馈膜的元素构成造成损坏。综上可以 看出, 紫外臭氧分解方法对去除 MCP 表面碳污染作 用明显。

\section{3 氢气热处理试验}

氢气热处理防离子反馈 $\mathrm{MCP}$ 是一种全新的分解 有机膜技术, 即在高温氢气环境中, 借助大量 $\mathrm{H}$ 的参 与, 与有机膜中的 $\mathrm{C}$ 原子通过化学作用生成易挥发的 小分子碳氢化合物, 从而达到去除 $\mathrm{C}$ 污染的目的。对 样品进行 $400^{\circ} \mathrm{C}$, 保温 $1 \mathrm{~h}$ 氢气热处理后进行测试, 结果如表 3 所示。

由表 3 可以看出, 防离子反馈 MCP 经过烧氢处 理后, 增益增加十分显著, 提升幅度接近 $30 \%$, 一方 面说明氢气热处理分解有机膜效果较好, 次级电子发 射体表面的 C 污染得到有效去除, 通道内表面二次电 子发射系数变大, 增益提高; 另外, 得益于氢气的强

烈还原作用使 MCP 通道壁内表面附着的氧化物还原 分解, MCP 表面得到净化, 通道壁表面势垒降低, 增 益得到提高, 这点从面电阻、体电阻都有不同程度的 降低可以得到验证。图 5 是试验后样品深度剖面的 AES 成分分布。

从图 5 中可以看出, 经氢气处理后的防离子反馈 $\mathrm{MCP}$ 蚀刻时间为 0 时, $\mathrm{C}$ 元素含量为 $20 \%$ 左右; 之后 迅速减少, 当蚀刻进行到 $10 \mathrm{~s}$ 时 $\mathrm{C}$ 元素降低至 5\%以 下, $30 \mathrm{~s}$ 左右 $\mathrm{C}$ 元素消失, 可见, $\mathrm{C}$ 元素只存在于防 离子反馈膜的表面, 并且不会对 MCP 表面的防离子 反馈膜的元素构成造成破坏。说明在氢气的作用下, 有机膜分解得较为彻底, 仅有微量 $\mathrm{C}$ 元素存在于防离 子反馈膜的表面。

\section{4 不同试验结果的讨论分析}

图 6 为长时间真空烘烤 $\left(400^{\circ} \mathrm{C}, 15 \mathrm{~h}\right)$ 、紫外臭 氧辐照 $50 \mathrm{~h}$ 及氢气热处理 $\left(400^{\circ} \mathrm{C}, 1 \mathrm{~h}\right)$ 的 $\mathrm{C}$ 元素深 度分布测试结果对比。3 个样品初始的 C 元素含量虽 有差别, 但对分析不同试验下 $\mathrm{C}$ 元素含量的客观变化 趋势影响不大。

从图 6 可以看出, 在紫外臭氧辐照和氢气热处理 试验中, 蚀刻 $30 \mathrm{~s}$ 之后, $\mathrm{C}$ 元素含量都趋近于 $0, \mathrm{C}$ 元素仅仅存在防离子反馈膜层表面, 去除 $\mathrm{C}$ 污染效果 显著。而真空条件下, 蚀刻 $10 \mathrm{~s}$ 之后, $\mathrm{C}$ 元素含量一 直在 $10 \%$ 左右小幅变动, 增加烘烤时间对 C 元素的分 解影响甚微, MCP 表面 $\mathrm{C}$ 残留严重, 即有机膜不能

\section{表 3 氢气热处理 $\mathrm{MCP}$ 参数测试结果}

Table 3 MCP test results after hydrogen heating process

\begin{tabular}{|c|c|c|c|c|c|c|}
\hline \multirow[b]{2}{*}{ Number } & \multicolumn{2}{|c|}{$R_{\mathrm{a}} / \mathrm{M} \Omega$} & \multicolumn{2}{|c|}{$R_{\mathrm{b}} /(800 \mathrm{~V}) / \mathrm{M} \Omega$} & \multicolumn{2}{|c|}{$G(800 \mathrm{~V})$} \\
\hline & $\begin{array}{c}\text { Before } \\
\text { processing }\end{array}$ & $\begin{array}{c}\text { After } \\
\text { processing }\end{array}$ & $\begin{array}{c}\text { Before } \\
\text { processing }\end{array}$ & $\begin{array}{c}\text { After } \\
\text { processing }\end{array}$ & $\begin{array}{c}\text { Before } \\
\text { processing }\end{array}$ & $\begin{array}{c}\text { After } \\
\text { processing }\end{array}$ \\
\hline $11 \#$ & 64 & 55 & 125 & 109 & 1236 & 1597 \\
\hline $12 \#$ & 77 & 59 & 130 & 118 & 1569 & 2012 \\
\hline
\end{tabular}




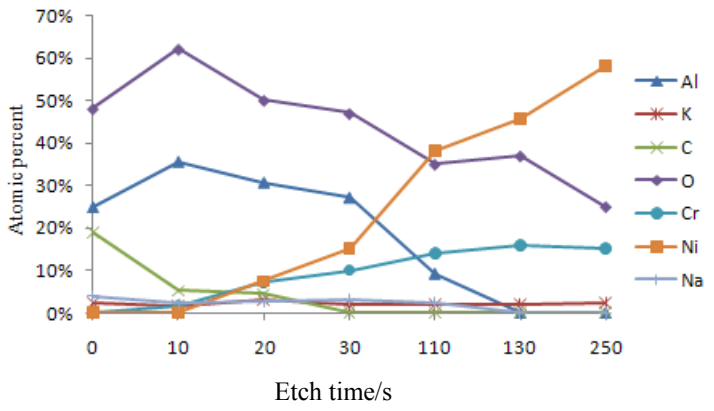

图 5 氢气热处理 MCP 深度剖面分析

Fig.5 Analysis of MCP depth profiles after hydrogen heating process

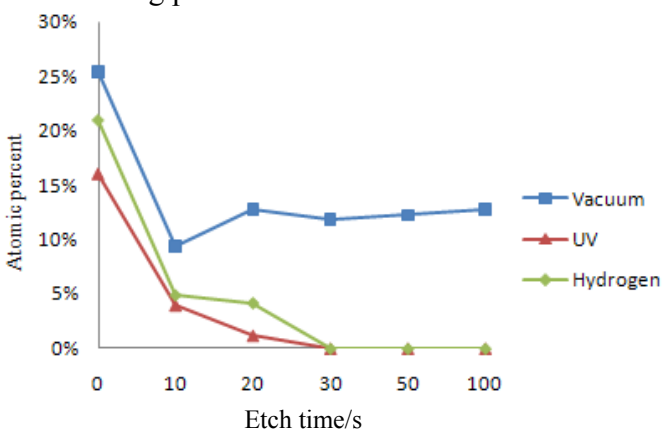

图 6 不同试验条件的 $\mathrm{MCP}$ 深度剖面 $\mathrm{C}$ 元素含量分析

Fig.6 C element analysis of MCP depth profiles at different test conditions

进行彻底分解。有机膜在真空中的分解反应过程如式 (1)所示:

$$
\begin{gathered}
2\left[\mathrm{C}_{6} \mathrm{H}_{7} \mathrm{O}_{2}\left(\mathrm{ONO}_{2}\right)_{3}\right] \mathrm{n}=6 \mathrm{nCO}_{2} \uparrow+2 \mathrm{nNO} \uparrow+2 \mathrm{nN}_{2} \uparrow+ \\
2 \mathrm{nCH}_{4} \uparrow+3 \mathrm{nH}_{2} \mathrm{O} \uparrow+5 \mathrm{nCO} \uparrow+\mathrm{nC} \text { (残留物) }
\end{gathered}
$$

从式(1)中可以看出, 真空条件下由于 $\mathrm{O} 、 \mathrm{H}$ 等活 泼元素的缺乏, 部分 $\mathrm{C}$ 元素不能与其形成易挥发性化 合物进行脱附, 从而导致分解物中有大量的 C 残留存 在。而在紫外臭氧辐照及氢气气氛条件下, 借助于初 生态 $\left[\mathrm{O}\right.$ 和大量 $\mathrm{H}$ 元素的参与, 与 $\mathrm{C}$ 形成 $\mathrm{CO}_{2} 、 \mathrm{C}-\mathrm{H}$ 化合物逸出 MCP 表面, 因此有机膜的分解较为彻底, $\mathrm{C}$ 污染现象可以得到根除。另外, 从 MCP 增益的变 化及时间成本上对 3 组试验进行对比可知, 氢气热处 理对 MCP 的增益提升巨大, 且耗时最少, 紫外臭氧 辐照虽可以对增益有一定的提升, 但耗时较长, 工艺 生产中效率太低, 而真空热处理时间的增加对 MCP 增益的提升几乎没有作用。

\section{4 结论}

本文研究了真空热处理、紫外臭氧辐照及氢气热 处理防离子反馈 MCP 试验, 得到的结论主要如下:

1) $\mathrm{C}$ 含量的降低对 MCP 性能的提升尤其是增益 的提升作用显著。

2) 紫外臭氧辐照和氢气热处理均能使 MCP 表面 $\mathrm{C}$ 残留分解较为彻底, 去除 $\mathrm{C}$ 污染效果显著。而真空 条件下 $\mathrm{C}$ 元素不能进行彻底分解, C 残留明显。
3 ) 氢气热处理对 MCP 的增益提升最大且效率较 高, 紫外臭氧辐照在 $50 \mathrm{~h}$ 后增益提升较为明显, 但效 率较低, 不适宜大批量工艺生产。

\section{参考文献:}

[1] 向世明, 倪国强. 光电子成像器件原理[M]. 北京: 国防工业出版社, 1999.

XIANG Shiming, NI Guoqiang. Principles of Photoelectronic Imaging Devices[M]. Beijing: National Defence Industry Press, 1999.

[2] 李晓峰, 张景文, 高鸿楷, 等. 三代管 MCP 离子阻挡膜研究[J]. 光子 学报, 2001, 30(12): 1496-1497.

LI Xiaofeng, ZHANG Jingwen, GAO Hongkai, et al. Ion barrier of MCP in the third generation image intensifier[J]. Acta Photonica Sinica, 2001, 30(12): 1496-1497.

[3] 张太民, 石峰, 朱宇峰, 等. 电子束蒸镀 MCP 超薄防离子反馈膜及膜 层特性分析[J]. 应用光学, 2012, 33(6): 1113-1115.

ZHANG Taimin, SHI Feng, ZHU Yufeng, et al. Preparation of ion barrier film of MCP with e-beam evaporation and its properties analysis[J]. Journal of Applied Optics, 2012, 33(6): 1113-1115.

[4] Pollehn H K. Performance and reliability of third generation image intensifiers[J]. Advances in Electronics and Electron Physics, 1985, 64A: 61-67.

[5] 间金良. 微通道板离子阻挡膜[J]. 应用光学, 1996, 17(1): 13 -14. YAN Jinliang. Microchannel plate ion barrier film[J]. Journal of Applied Optics, 1996, 17(1): 13 -14.

[6]间金良, 向世明. 微通道板离子阻挡膜对III代像管分辨率的影响 [J]. 应用光学, 1997, 18(6): 24 -30.

YAN Jinliang, XIANG Shiming. Effect of ion barrier film at the input of $\mathrm{MCP}$ on the resolution of generation III image tube[J]. Journal of Applied Optics, 1997, 18(6): 24 -30.

[7] Korobov M I. Passage of electrons through a dielectric film microchannel plate structure[J]. Soviet Journal of Optical Technology, 1988, 55(5): 266 $-270$

[8] 田景全, 姜德龙, 富丽晨, 等. 微通道板非晶态 $\mathrm{Al}_{2} \mathrm{O}_{3}$ 电子透射膜 $[\mathrm{J}]$. 电子学报, 1996, 24(8): 1-5.

TIAN Jingquan, JIANG Delong, FU Lichen, et al. Amorphous $\mathrm{Al}_{2} \mathrm{O}_{3}$ electron-transmitting film on microchannel plate[J]. Chinese Journal of Electronics, 1996, 24(8): 1-5.

[9] 问金良. 带膜微通道板的工艺和特性研究[J]. 红外技术, 2003, 25(4): 84-85.

YAN Jinliang. Technology and performance of filmed microchannel plate[J]. Infrared Technology, 2003, 25(4): 84-85.

[10] 孙忠文, 黄永刚, 贾金升, 等. 酸蚀对微通道板电性能的影响[J]. 应 用光学, 2008, 29(2): 161-165.

SUN Zhongwen, HUANG Yonggang, JIA Jinsheng, et al. Effect of acid etching on electrical performances of microchannel plate[J]. Journal of Applied Optics, 2008, 29(2): 161-165.

[11] 间金良, 赵银女. 紫外光辐照清洗带膜 MCP 的工艺研究[J]. 应用光 学, 1998, 19(6): 38-39.

YAN Jinliang, ZHAO Yinnu. Cleaning of filmed microchannel plate by ultraviolet radiation[J]. Journal of Applied Optics, 1998, 19(6): 38-39. 